\title{
FINITE GENERATION OF RECURSIVELY ENUMERABLE SETS
}

\author{
JULIA ROBINSON
}

Suppose we wish to build up the class of recursively enumerable sets by starting with the set $\mathscr{N}$ of natural numbers and constructing new sets from those already obtained using as little auxiliary machinery as possible. One way would be to start with a finite number of functions $F_{1}, \cdots, F_{k}$ (of one variable, from and to $\Re$ ) such that every recursively enumerable set can be obtained from $\mathfrak{x}$ by constructing new sets $F_{j}[J]$ where $J$ is a previously obtained set. We can think of $F_{1}, \cdots, F_{k}$ as unary operations on sets of natural numbers. Any set $S$ obtained in this way is the range of a function $F$ obtained by composition from $F_{1}, \cdots, F_{k}$. If we consider the values of $F_{1}, \cdots, F_{k}$ as given, then the number of steps needed to compute $F n$ does not depend on $n$. Hence for all $x \in \mathcal{S}$, there exists a proof that $x \in \mathcal{S}$ of bounded length in terms of $F_{1}, \cdots, F_{k}$ (just as there is a one-step proof that a composite number is composite in terms of multiplication).

We say a set of natural numbers is generated by $F_{1}, \cdots, F_{k}$ if it is the range of a function obtained by composition from $F_{1}, \cdots, F_{k}$. Also a class $\mathcal{C}$ of sets is generated by $F_{1}, \cdots, F_{k}$ if every nonempty set of $\mathcal{C}$ is generated by $F_{1}, \cdots, F_{k}$ and every set generated by $F_{1}, \cdots, F_{k}$ is in $\mathcal{e}$.

Example. Let $G_{0}, G_{1}, \cdots$ be the primitive recursive functions listed systematically so that the function $G$ given by

$$
G\left(2^{n}(2 x+1)-1\right)=G_{n} x
$$

is recursive. Then

$$
G_{n}=G(S D)^{n} D
$$

where $S x=x+1$ and $D x=2 x$. Every nonempty recursively enumerable set is the range of some primitive recursive function and hence the range of $G(S D)^{n} D$ for some $n$. Since $S, D$, and $G$ are recursive functions, any function obtained from them by composition will be recursive and will have a recursively enumerable range. Thus $G, S$, and $D$ generate the class of recursively enumerable sets. However, they do not form an interesting set of generators since all the work of listing recursively enumerable sets is done in computing $G$.

Received by the editors August 22, 1967. 
In this paper, we give sets of easy-to-compute generators for the classes of recursively enumerable sets and diophantine sets, i.e. sets which are existentially definable in terms of + and $\cdot$. General theorems describing classes of sets which can be finitely generated are proved in [3].

Let $I x=x, O x=0, T x=2^{x}, Z x=0^{x}$, and $E(x, y)$ be the characteristic function of equality, i.e. $E(x, y)=0^{|x-y|}$. We shall also use pairing functions. If $J(x, y)$ maps the set of ordered pairs of natural numbers onto $\mathscr{T}$ in a one-to-one way, then $J$ and its inverse functions $K$ and $L$, given by $K J(x, y)=x$ and $L J(x, y)=y$, are called pairing functions. We shall always take $J$ to be the Cantor pairing function given by

$$
J(x, y)=\frac{1}{2}\left((x+y)^{2}+3 x+y\right)
$$

unless otherwise stated. Its inverse functions are probably the simplest functions which assume every natural number infinitely often. They can easily be computed recursively by the equations

$$
\begin{aligned}
& K 0=L 0=0, \\
& K(x+1)=0, \quad L(x+1)=K x+1 \quad \text { if } L x=0, \\
& K(x+1)=K x+1, \quad L(x+1)=L x-1 \quad \text { if } L x \neq 0 .
\end{aligned}
$$

Example. Let $J, K$, and $L$ be any pairing functions and let $G$ be given by $G(J(x, n))=G_{n} x$. Then $G_{n} x=G J(K, S L)^{n} J(I, O) x$. In the example above, we took $J(x, n)=2^{n}(2 x+1)-1$, so $J(x, n+1)$ $=2 J(x, n)+1$ and $J(x, 0)=2 x$.

Lemma 1. The functions $O x, Z x, x+y, x \cdot y$, and $E(x, y)$ are all obtainable from $J, K, S$, and $D$ by substitution.

Proof. R. M. Robinson [4, p. 665], derived the remarkable identity for all $x$ and $y$ with $x \geqq y$ :

$$
x-y=K D D J(S S S K D D J(S S S K D D J(y, D D x), x), D y) .
$$

He then used it to define $x+y$ by substitution from $J, K, S$, and $D$. We shall let $x-y$ denote the function on the right of (2) for all $x$ and $y$. Then $O x=x-x$ and $x \cdot y=(J(0, x+y)-J(0, x))-J(0, y)$. To define $Z$, we make use of (1) to see that

$$
S J(0,0)=J(0,1), \quad S J(0, x+1)=J(1, x) .
$$

Hence $K S J(0, x)=\operatorname{sgn} x$. Since $K 2=1$ and $K 3=0$, we have $Z x$ $=K S S K S J(0, x)$. Finally, $E(x, y)=Z((x-y)+(y-x))$.

THEOREM 1. Every nonempty recursively enumerable set is the range of a function of one variable obtained from $S, D, T, K$, and $L$ by com- 
position and pairing. Conversely, the range of such a function is recursively enumerable. If $T$ is omitted from the set of initial functions then just the nonempty diophantine sets are obtained.

Proof. Every recursively enumerable set $\delta$ is exponential diophantine and conversely. (See Davis, Putnam, and Robinson [1].) Hence

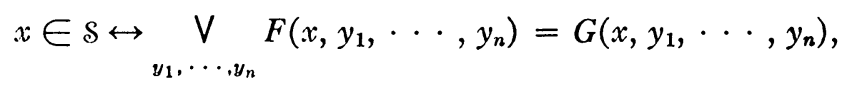

where $F$ and $G$ are suitably chosen terms built up from $x, y_{1}, \cdots, y_{n}$, and particular natural numbers by means of,$+ \cdot$, and $T$. (See $[1$, Corollary 5].) Let $a$ be any element of $\mathcal{S}$. Then $\mathcal{S}=\mathbb{R}(H)$ where

$$
\begin{aligned}
H\left(x, y_{1}, \cdots, y_{n}\right)= & x \cdot E\left(F\left(x, y_{1}, \cdots, y_{n}\right), G\left(x, y_{1}, \cdots, y_{n}\right)\right) \\
& +a \cdot Z E\left(F\left(x, y_{1}, \cdots, y_{n}\right), G\left(x, y_{1}, \cdots, y_{n}\right)\right) .
\end{aligned}
$$

Let $M=H\left(K, K L, \cdots, K L^{n-1}, L^{n}\right)$. Then $M$ is a function of one variable obtained from $O, S, K, L, T$, and $Z$ by forming new functions $F$ from previously obtained functions $A$ and $B$ by taking $F=A B$, $F=A+B, F=A \cdot B$, and $F=E(A, B)$. Also $R(M)=R(H)=\delta$. By Lemma 1 , we see that every such function $M$ can be obtained from $K, L, S, D, T$, and $I=J(K, L)$ by composition and pairing. On the other hand, $M$ is primitive recursive so $R(M)$ is recursively enumerable.

The proof that every nonempty diophantine set is obtained if we omit $T$ from the set of initial functions, is obtained from the above proof by omitting $T$ throughout. A function $F$ is said to be diophantine if there is a polynomial $P$ with integer coefficients such that

$$
y=F x \leftrightarrow{ }_{u_{1}, \cdots, u_{n}} P\left(x, y, u_{1}, \cdots, u_{n}\right)=0 .
$$

Hence if $F$ is diophantine then $R(F)$ is a diophantine set. Indeed,

$$
y \in R(F) \leftrightarrow \bigvee_{x, u_{1}, \cdots, u_{n}} P\left(x, y, u_{1}, \cdots, u_{n}\right)=0 .
$$

Also, if $F$ and $G$ are diophantine then $F G$ is diophantine. Clearly, $J, K, L, S$, and $D$ are diophantine functions so the range of any function obtained from $K, L, S$, and $D$ by composition and pairing is diophantine.

REMARK. It is not known whether all recursively enumerable sets are diophantine. Hence we do not know whether $T$ is necessary to obtain all recursively enumerable sets.

Let $C=J(K L, J(L L, K))$. Then $C^{3}=I$, since $C J(x, J(y, z))$ $=J(y, J(z, x))$. For any function $A$, we define $A^{*}=J(K, A L)$. Then for all $A$ and $B$ 


$$
\begin{aligned}
(A B)^{*} & =A^{*} B^{*}, \\
J(A, B) & =B^{*} J(L, K) A^{*} J(I, I), \\
A^{* *} & =C J(L, K) A^{*} J(L, K) C^{2} .
\end{aligned}
$$

These formulas can be easily checked by carrying out the compositions indicated on the right using the fact that $J(F K, G L) J(M, N)$ $=J(F M, G N)$ and $J(F L, G K) J(M, N)=J(F N, G M)$ etc.

Lemma 2. If $F$ can be obtained from $A_{1}, \cdots, A_{n}, K$, and $L$ by composition and pairing, then $F$ can be obtained from $A_{1}{ }^{*}, \cdots, A_{n}{ }^{*}, K$, $J(L, K), J(I, I)$, and $J(K L, J(L L, K))$ by composition alone. Here $J$, $K$, and $L$ can be arbitrary pairing functions.

Proof. Let $Q$ be the least class of functions closed under composition which contains $A_{1}{ }^{*}, \cdots, A_{n}{ }^{*}, K, J(L, K), J(I, I)$, and $C$. We wish to show that if $F$ is obtained from $A_{1}, \cdots, A_{n}, K$, and $L$ by composition and pairing, then $F$ belongs to $a$. Since $F=L F^{*} J(I, I)$ and $L=K J(L, K)$, it is sufficient to show that $F^{*}$ belongs to $Q$, and this will be done by induction.

I. Suppose $F=A_{j}$ then $F^{*}$ belongs to $a$ by definition. Also $K^{*}$ $=L C^{2}$ and $L^{*}=J(L, K) L C$. Hence both $K^{*}$ and $L^{*}$ belong to $a$.

II. Suppose $F=A B$ where $A^{*}$ and $B^{*}$ belong to $Q$. Then $F^{*}=A^{*} B^{*}$ so $F^{*}$ belongs to $Q$.

III. Suppose $F=J(A, B)$ where $A^{*}$ and $B^{*}$ belong to $a$. Then $F=B^{*} J(L, K) A^{*} J(I, I)$.

Hence

$$
F^{*}=B^{* *} J(L, K)^{*} A^{* *} J(I, I)^{*} .
$$

By (5), $B^{* *}$ and $A^{* *}$ can be obtained by composition from $C, J(L, K)$, $B^{*}$, and $A^{*}$. Hence we need only show that $J(L, K)^{*}$ and $J(I, I)^{*}$ belong to $Q$. Now

$$
J(L, K)^{*}=J(K, J(L L, K L))=C J(K L, J(K, L L))=C J\left(K L, L^{*}\right) .
$$

Hence by (4),

$$
J(L, K)^{*}=C L^{* *} J(L, K) K^{*} L^{*} J(I, I) .
$$

Also

$$
J(I, I)^{*}=J(K, J(L, L))=L^{* *} C J(I, I) .
$$

Finally, $K^{* *}$ and $L^{* *}$ belong to $\propto$ by (5) and I, hence $F^{*}$ belongs to $Q$.

THeOREM 2. The class of recursively enumerable sets is generated by $K, J(K, S L), J(K, D L), J(K, T L), J(L, K), J(I, I), J(K L, J(L L, K))$. 
This set of generators with $J(K, T L)$ deleted generates the class of diophantine sets.

TheOREM 2 is an immediate consequence of Lemma 2 and Theorem 1.

REMARK. By the lemma on page 714 of [2], the functions $K$, $J(L, K)$, and $J(I, I)$ can be replaced by the two functions $J(L K, K L)$ and $J(L, I)$. (Recall that $K=J(K K, L K)$.) Indeed, the total number of generators can be reduced to two,

$$
K \text { and } J\left(L, J\left(F_{1}, J\left(F_{2}, \cdots, J\left(F_{n-1}, F_{n}\right)\right)\right) \cdots\right),
$$

where $F_{1}, \cdots, F_{n}$ are the generators other than $K$.

Lemma 3. If $F$ can be obtained by composition and pairing from $A_{1}, \cdots, A_{n}, K$, and $L$ then $F=K B$ for some function $B$ obtained by composition from $A_{1}^{*}, \cdots, A_{n}^{*}, J(L, K), J(I, I), J(K L, J(L L, K))$, $J(L, K)^{*}, J(I, I)^{*}$, and $J(K L, J(L L, K))^{*}$. Here $J, K$, and $L$ can be arbitrary pairing functions.

Proof. By Lemma 2,

$$
F=B_{0} K B_{1} K \cdots B_{t-1} K B_{t}
$$

for some $B_{0}, \cdots, B_{t}$ obtained from $A_{1}^{*}, \cdots, A_{n}^{*}, J(L, K), J(I, I)$, and $J(K L, J(L L, K))$ by composition. We can take $t>0$ since $B_{0}=B_{0} K J(I, I)$. (If $B_{i}$ is the identity function, then $B_{i}=J(L, K)^{2}$.) Now

$$
J(L, K) A^{*} J(L, K)=J(A K, L) .
$$

Hence $A K=K J(L, K) A^{*} J(L, K)$. Thus each $K$ in (6) can be brought to the front in turn. For example,

$$
\begin{aligned}
F & =B_{0} K B_{1} K \cdots \\
& =K J(L, K) B_{0}{ }^{*} J(L, K) B_{1} K \cdots \\
& =K^{2} J(L, K) J(L, K)^{*} B_{0}{ }^{* *} J(L, K)^{*} B_{1}{ }^{*} J(L, K) B_{2} K \cdots \\
& : \\
& =K^{t} H
\end{aligned}
$$

where $H$ is obtained from the functions listed in the lemma by composition. Finally,

$$
K K=K J(K L, J(L L, K)) \cdot J(L, K)=K C J(L, K)
$$




$$
F=K^{t} H=K(C J(L, K))^{t-1} H .
$$

THEOREM 3. Every nonempty recursively enumerable set is the range of a function $K B$ where $B$ is obtained by composition from

$$
S^{*}, \quad D^{*}, \quad T^{*}, J(L, K), \quad J(I, I), \quad C, \quad J(L, K)^{*}, J(I, I)^{*}, C^{*} .
$$

The range of $B$ is a primitive recursive set. If $T^{*}$ is deleted from the set of functions (7) then just the nonempty diophantine sets are obtained. In this case, both the range of $B$ and its complement are diophantine.

Proof. In light of Lemma 3 and Theorem 1, we need only show that $\mathbb{R}(B)$ is a primitive recursive set in the first case and $\mathcal{C} R(B)$ is diophantine in the second case. (Here $\mathcal{C} R(B)$ is the complement of the range of $B$.) Notice that $G x \geqq x$ for $G$ equal to $S^{*}, D^{*}, T^{*}$, $J(I, I)^{*}$, or $J(I, I)$. This is clear for the *-functions since $J(x, y)$ $\leqq J(x, z)$ whenever $y \leqq z$. Thus $S^{*} x=J(K, S L) x \geqq J(K, L) x=x$, etc. Also $J(I, I) x=J(x, x) \geqq x$. Hence if $G$ is one of these functions, then

$$
x \in R(G H) \leftrightarrow \bigvee_{y \leq x}(G y=x \wedge y \in R(H)) .
$$

Furthermore, if $G$ is one of the remaining functions of (7), then $G$ is a primitive recursive permutation such that $G^{-1}$ is also primitive recursive. Indeed, $C^{-1}=C^{2},\left(C^{*}\right)^{-1}=C^{*} C^{*}, J(L, K)^{-1}=J(L, K)$, and $\left(J(L, K)^{*}\right)^{-1}=J(L, K)^{*}$. Hence

$$
x \in \mathfrak{R}(G H) \leftrightarrow G^{-1} x \in R(H) .
$$

Therefore by induction starting with $H=I$, we see that $R(B)$ is primitive recursive.

In the diophantine case, all the functions of $(7)$ with $T^{*}$ excluded are diophantine. Hence if $B$ is obtained from them by composition, $R(B)$ is diophantine. If $M$ is strictly monotone, then

$$
\begin{aligned}
x \in \mathfrak{C} R(M) & \leftrightarrow \bigvee(M y<x<M(y+1)), \\
x \in \mathcal{V}\left(M^{*}\right) & \leftrightarrow \bigvee_{y}(M y=L x), \\
x \in \mathcal{C} R\left(M^{*}\right) & \leftrightarrow \bigvee_{y}(M y<L x<M(y+1)) .
\end{aligned}
$$

If $G$ is a univalent function, then

$$
\mathfrak{C} R(G H)=\mathbb{R}(G\lceil\mathfrak{C} \mathbb{R}(H)) \cup \mathfrak{C} R(G) .
$$

Suppose $\mathbb{R}(H)$ and $\mathcal{C} R(H)$ are diophantine, and $G$ is one of the functions $S^{*}, D^{*}, J(I, I)$, and $J(I, I)^{*}$. Then $\mathcal{e} R(G)$ is diophantine by (8) or (10). Hence by (11), 


$$
x \in \mathfrak{C} \mathfrak{R}(G H) \leftrightarrow \bigvee_{y}((y \in \mathfrak{C} \mathfrak{R}(H) \wedge G y=x) \bigvee x \in \mathfrak{C} R(G)),
$$

so $\mathbb{e} \mathfrak{R}(G H)$ is diophantine. If $G$ is one of the permutations of $(7)$, then

$$
x \in \mathfrak{C} \mathfrak{R}(G H) \leftrightarrow \bigvee_{y}(G y=x \wedge y \in \mathfrak{e} \mathfrak{R}(H)) .
$$

Hence by induction $\mathcal{C} R(B)$ is diophantine.

\section{REFERENCES}

1. Martin Davis, Hilary Putnam and Julia Robinson, The decision problem for exponential diophantine equations, Ann. of Math. 74 (1961), 425-436.

2. Julia Robinson, General recursive functions, Proc. Amer. Math. Soc. 1 (1950), 703-718.

3. - Finitely generated classes of sets, Proc. Amer. Math. Soc. (to appear).

4. Raphael M. Robinson, Primitive recusive functions. II, Proc. Amer. Math. Soc. 6 (1955), 663-666.

University of California, Berkeley 